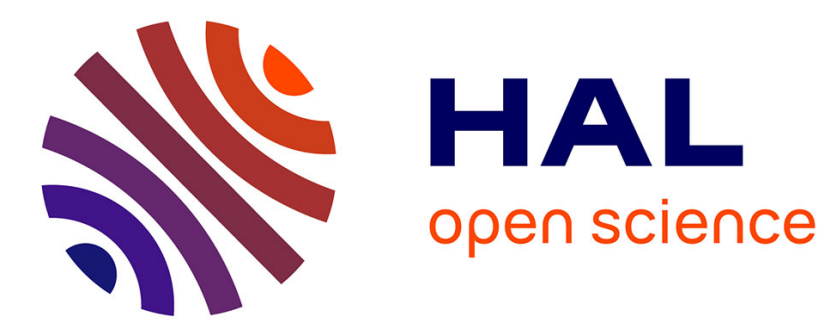

\title{
Slice-to-Volume Deformable Registration: Efficient one shot consensus between plane selection and in-plane deformation
}

\author{
Enzo Ferrante, Vivien Fecamp, Nikos Paragios
}

\section{- To cite this version:}

Enzo Ferrante, Vivien Fecamp, Nikos Paragios. Slice-to-Volume Deformable Registration: Efficient one shot consensus between plane selection and in-plane deformation. International Journal of Computer Assisted Radiology and Surgery, 2015, 10 (6), pp.791-800. 10.1007/s11548-015-1205-2 . hal$01118871 \mathrm{v} 2$

\section{HAL Id: hal-01118871 \\ https://hal.inria.fr/hal-01118871v2}

Submitted on 16 Jun 2015

HAL is a multi-disciplinary open access archive for the deposit and dissemination of scientific research documents, whether they are published or not. The documents may come from teaching and research institutions in France or abroad, or from public or private research centers.
L'archive ouverte pluridisciplinaire HAL, est destinée au dépôt et à la diffusion de documents scientifiques de niveau recherche, publiés ou non, émanant des établissements d'enseignement et de recherche français ou étrangers, des laboratoires publics ou privés. 


\title{
Slice-to-Volume Deformable Registration
}

\section{Efficient one shot consensus between plane selection and in-plane deformation}

\author{
Enzo Ferrante • Vivien Fecamp · Nikos \\ Paragios
}

Received: date / Accepted: date

\begin{abstract}
Purpose: This paper introduces a novel decomposed graphical model to deal with slice-to-volume registration in the context of medical images and image guided surgeries.

Methods: We present a new non-rigid slice-to-volume registration method whose main contribution is the ability to decouple the plane selection and the in-plane deformation parts of the transformation - through two distinct graphs - towards reducing the complexity of the model while being able to obtain simultaneously the solution for both of them. To this end, the plane selection process is expressed as a local graph-labeling problem endowed with planarity satisfaction constraints, which is then directly linked with the deformable part through the data registration likelihoods. The resulting model is modular with respect to the image metric, can cope with arbitrary in-plane regularization terms and inherits excellent properties in terms of computational efficiency.

Results: The proof of concept for the proposed formulation is done using cardiac MR sequences of a beating heart (an artificially generated 2D temporal sequence is extracted using real data with known ground truth) as well as multimodal brain images involving ultrasound and computed tomography images. We achieve state of the art results while decreasing the computational time when we compare with another method based on similar techniques.

Conclusions: We confirm that graphical models and discrete optimization techniques are suitable to solve non-rigid slice-to-volume registration problems. Moreover, we show that decoupling the graphical model and labeling it using two lower dimensional label spaces, we can achieve state of the art results while substantially reducing the complexity of our method and moving the
\end{abstract}

Center for Visual Computing (CVN), CentraleSupelec — Galen Team, INRIA

92295 Chatenay-Malabry, France

Tel.: (+33) 0141131630

E-mail: enzo.ferrante@ecp.fr 
approach close to real clinical applications once considered in the context of modern parallel architectures.

Keywords Slice-to-Volume Registration · 2D-3D Registration · Discrete Optimization · Graphical Models · Markov Random Fields

\section{Introduction}

The problem of slice-to-volume deformable image registration consists in aligning a sliced 2D image (e.g. Ultrasound or US) to its corresponding plane from a 3D volume (e.g. Computer Tomography or CT). We call it deformable registration because the $2 \mathrm{D}$ image can be deformed during the registration process.

This problem finds applications in many medical image related contexts such as computer aided-biopsy [19], motion correction for image reconstruction [5], tumor ablation [22] and image-guided surgery (IGS) [23]. In the case of image guided procedures, a pre-operative 3D image and several intra-operative $2 \mathrm{D}$ acquisitions are to be fused towards providing position and navigation information to the surgeons. Nowadays, this fusion is mainly performed using two different tracking technologies: optical (OTS) and electromagnetic (EMTS) tracking systems. In the first case, OTS requires a line-of-sight to be maintained between the tracking device and the instrument to be tracked; this fact can disturb doctors during their work and is not always convenient. In the second case, EMTS does not have line-of-sight requirements but it is very susceptible to distortion from nearby metal sources and presents limited accuracy compared to optical tracking [4]. Moreover, nor OTS neither EMTS can deal with deformations between intra and pre-operative images. In this work, we propose to use 2D-3D slice-to-volume registration algorithms which are purely image based to solve this challenging problem and overcome the limitations presented by current technologies.

The problem of deformable image registration has been a pillar of computer vision (optical flow) and medical imaging (image fusion), and therefore one can cite numerous methods to perform 2D-2D and 3D-3D registration [11] [1]. However, the problem of 2D-3D registration, and particularly the problem of slice-to-volume registration, deserves separate investigation and specific methods development. While a single 2D slice contains less information than a $3 \mathrm{D}$ volume, the solution remains a 3D mapping function (a deformation field in case of non-rigid registration or a transformation matrix in case of rigid registration) as in the case of $3 \mathrm{D}-3 \mathrm{D}$ registration. This fact converts $2 \mathrm{D}$ to $3 \mathrm{D}$ slice-to-volume registration in a really challenging problem. The other case of $2 \mathrm{D}-3 \mathrm{D}$ registration problems, where projective $2 \mathrm{D}$ images such as X-Ray images are registered with volumetric images (CT for example) has received more attention in the last years [18] [15] and is not covered in this paper.

A variety of methods has been proposed to deal with slice-to-volume registration. In [3], standard optimization approaches and heuristics (as Simplex and Simulated Annealing algorithms) are applied on FluroCT to CT registration, testing with different intensity based similarity measures. [6] presents 
a feature based method that performs slice-to-volume registration, using several slices in order to improve the quality of the results. [10] tracks intraoperative MRI slices of prostate images with a pre-operative MRI volume. This monomodal registration (MRI intra-operative slices to MRI pre-operative volume) is designed to provide patient tracking information for prostate biopsy performed under MR guidance. A similar problem is tackled by [25] where a two-step algorithm (rigid registration in the first step, and deformable registration in the second one) is applied to register three orthogonal intra-operative MR slices with a pre-operative volume. [23] proposes a method to register endoscopic and laparoscopic US images with pre-operative CT volumes. It is based on a new phase correlation technique called LEPART and it manages only rigid registration in quasi real time. [21] presents a flexible framework for intensity based slice-to-volume non-rigid registration algorithms that was used to register histological sections images to MRI of the human brain.

The main limitations of the aforementioned methods are their specificity to the clinical context (they are derived and can be used for specific clinical applications), the requirement of anatomical segmentations in some of them that increases their complexity and often their sequential nature where first plane is selected and then in-plane deformation is determined. Graphical models are powerful formalisms that could be amended to overcome these limitations. Casting computer vision problems as labeling ones through the use of Markov Random Field (MRF) theory has gained attention since [9]. It has been widely used to solve non-rigid image registration in the last years [11] [16] [17], mainly for 2D-2D or 3D-3D. In [26], a method based on MRFs to perform 2D-3D registration is presented, but it estimates just rigid transformations and works with projective images. Regarding slice-to-volume registration using MRF, our previous work [7] presents a MRF framework based on a high dimensional label space to solve this problem; we will refer to it as the overparameterized method.

In this work, our aim is to introduce a low rank graphical model that is able to simultaneously perform plane selection and estimate the in-plane deformation between the 2D source image and the corresponding slice from the $3 \mathrm{D}$ volume. We decouple a physical control point of a regular grid in two nodes of the MRF graph, one taking labels from the plane selection label space and the other one from the in-plane deformations label space. In that way, the complexity of the model reduces to the square of the cardinality of the biggest label space (instead of being quadratic in the product of the cardinalities of the two spaces), with a slight increase of the graphical model connectivity. This technique has been previously applied in 2D-2D registration [24]. The main advantage is related to the fact that, while the number of nodes augment linearly, the number of labels is decreased in a quadratic order.

The main contributions of our paper with respect to our previous work [7] are therefore two-fold. Firstly, we propose a new way of decoupling the plane selection and the in-plane deformation label spaces towards a novel low rank model of order 3 (instead of a model of order 5 as in [7]); it results into a more tractable problem in terms of getting the optimal solution. Secondly, 


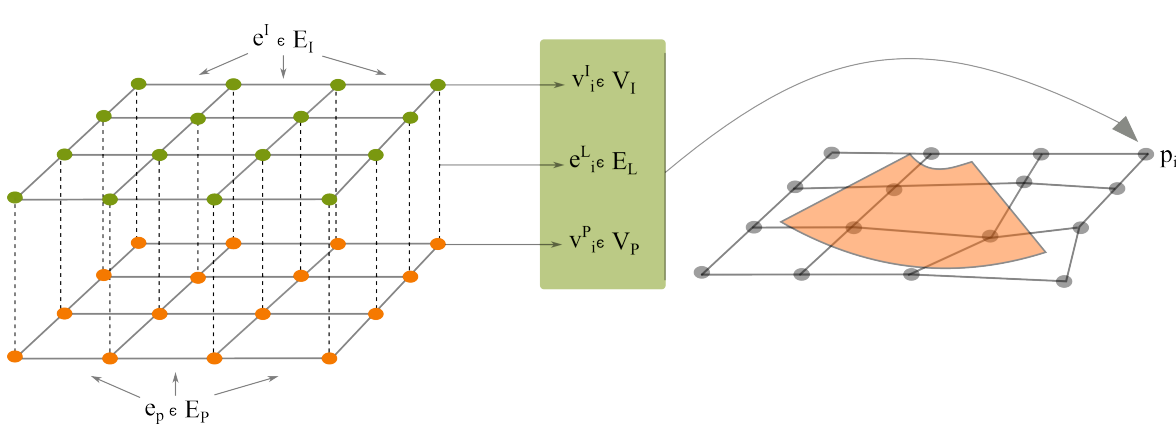

Fig. 1 Structure of the decoupled graph. The green nodes (top grid) are included in $V_{I}$ and orange ones (bottom grid) in $V_{P}$ modeling in-plane deformations and plane position respectively. Edges connecting $V_{I}$ nodes are part of $E_{I}$ and those connecting $V_{P}$ nodes are part of $E_{P}$; they are associated with regularization terms. Dotted lines represent cliques in $E_{D}$ that encode the matching similarity measure. Using this information we can reconstruct a deformed grid that is interpreted as a Free Form Deformation model. In the image we can appreciate how we associate two nodes of the graph with one control point of the grid.

we obtain substantial decrease of the search space size (order of 10), allowing much richer sampling of the label space, thus in theory more precise solutions. Moreover, by decoupling the label spaces it is possible to explore both of them with different sparseness levels.

The framework is intensity based and independent of the similarity measure, so it can be adapted to different image modalities or new measures. We tested our approach on two different datasets: a monomodal dataset where 2D MRI images of the heart are registered with MRI volumes, and another multimodal dataset where 2D US images are fused with CT volumes [20]. Both datasets were also used in [7].

The paper is organized as follows: in Section 2 we present the decoupled MRF formulation together with a complete explanation about the label spaces and the energy terms. In Section 3, the validation tests and results are presented and discussed. Finally, Section 4 concludes our paper and provides some ideas on relevant future directions.

\section{Method Description}

Non-rigid slice-to-volume registration can be seen as an optimization problem. We aim at optimizing an energy function by choosing the optimal plane (slice) $\hat{\pi}[J]$ from target volume $J$ and the optimal deformation field $\hat{T}_{D}$ as indicates the following equation:

$$
\hat{T}_{D}, \hat{\pi}=\underset{T_{D}, \pi}{\operatorname{argmin}} \mathcal{D}\left(I \circ T_{D}(\boldsymbol{x}), \pi[J](\boldsymbol{x})\right)+\mathcal{R}\left(T_{D}, \pi\right),
$$

where $I$ is the source $2 \mathrm{D}$ image, $\mathcal{D}$ represents the data term and $\mathcal{R}$ the regularization term. Given the 2D source image $I$ and the 3D target volume $J$, we seek the slice $\hat{\pi}[J]$ from volume $J$ that best matches the image $I$. We call 
it non-rigid registration because image $I$ can be deformed by the deformation field $\hat{T}_{D}$. The data term $\mathcal{D}$ measures the similarity between the source and the target, while the regularization term imposes smoothness constraints on the solution.

From this general optimization problem, we can derive different formulations. In [7], we proposed a high-dimensional label space based approach considering local labels of dimension five (plane + in-plane deformations). One of the main problems related to this high dimensionality is its consequently high computational cost. In this work, we try to avoid this problem by decoupling the label space in two different ones and reforming the structure of the graph to still capture rigid plane displacements and in-plane deformation.

Our formulation consists in an undirected pairwise graph $G_{D}=<V, E>$ with a set of nodes $V=V_{I} \cup V_{P}$ and a set of edges $E=E_{I} \cup E_{P} \cup E_{D} . V_{I}$ and $V_{P}$ have a 4-neighbor grid structure and the same cardinality. Nodes in $V_{I}$ are labeled with in-plane deformation labels, while labels used in $V_{P}$ represent the plane position. Edges from $E_{I}$ and $E_{P}$ correspond to a conventional pairwise neighborhood connection system for nodes in $V_{I}$ and $V_{P}$ respectively; they are associated with regularization terms $\left(E_{I}\right.$ corresponds to in-plane deformation regularizers and $E_{P}$ to the plane selection regularizers). Edges in $E_{D}$ link every node from $V_{I}$ to its corresponding node from $V_{P}$, creating a graph with a sort of three dimensional structure (see Figure 1); those terms associated to $E_{D}$ encode the data terms (i.e. the similarity measure).

In order to get a better understanding of the model, we can think of a single hypothetical grid similar to the one defined in [7], where every control point $\boldsymbol{p}_{\boldsymbol{k}}$ from this grid is associated with two nodes from our approach, i.e. $v_{k}^{I} \in V_{I}$ and $v_{k}^{P} \in V_{P}$. This idea is depicted in Figure 1 and it will be useful to understand the energy terms.

\section{Label Space}

We define two different label spaces, one associated with nodes in $V_{I}$ (called $\left.L_{I}\right)$ and the other one associated with nodes in $V_{P}$ (called $\left.L_{P}\right)$.

The first label space, $L_{I}$, is a bidimensional space that models in-plane deformation using displacement vectors $\boldsymbol{l}^{\boldsymbol{I}} \in E_{I}=\left(d_{x}, d_{y}\right)$.

The second label space, $L_{P}$, indicates the plane in which the corresponding control point is located. It consists of labels $\boldsymbol{l}^{\boldsymbol{P}}$ associated to different planes. In order to specify the plane and the orientation of the grid on it, we store an orthonormal basis of this plane together with the position of a reference point in this plane. Using this information, we can reconstruct the position of the rest of the control points in the grid. This way of storing the planes, allow us to implement different plane space sampling methods. In this work, we chose a simple uniformly sampling around the current plane position, varying rotation and translation parameters in a given range. This is an important advantage of our method: we could use prior knowledge to improve the way we explore the plane space, just by changing the plane space sampling method. 
To compute the final position of a control point we use both labels. First, the corresponding label in $L_{P}$ defines a $3 \mathrm{D}$ point belonging to a plane space with a given basis. Then, we use the corresponding label in $L_{I}$ to move the point in the $2 \mathrm{D}$ plane thanks to its basis.

\section{Objective Function}

The energy that guides the optimization process is defined on the pairwise terms. Two types of edges represent regularization terms while the last one represents the data terms; the energy is thus defined as:

$\mathcal{E}(I, P, D)=\min \left\{\gamma \sum_{(i, j) \in E_{I}} e_{i, j}^{I}\left(\boldsymbol{l}_{\boldsymbol{i}}^{\boldsymbol{I}}, \boldsymbol{l}_{\boldsymbol{j}}^{\boldsymbol{I}}\right)+\alpha \sum_{(i, j) \in E_{P}} e_{i, j}^{P}\left(\boldsymbol{l}_{\boldsymbol{i}}^{\boldsymbol{P}}, \boldsymbol{l}_{\boldsymbol{j}}^{\boldsymbol{P}}\right)+\beta \sum_{(i, j) \in E_{D}} e_{i, j}^{D}\left(\boldsymbol{l}_{\boldsymbol{i}}^{\boldsymbol{I}}, \boldsymbol{l}_{\boldsymbol{j}}^{\boldsymbol{P}}\right)\right\}$,

where $\gamma, \alpha$ and $\beta$ are positive weighting factors, $e_{i, j}^{I} \in I$ are the in-plane regularizers (associated to edges in $E^{I}$ ), $e_{i, j}^{P} \in P$ are the plane regularizers (associated with edges in $E^{P}$ ) and $e_{i, j}^{D} \in D$ the data terms (associated with edges in $\left.E^{D}\right) . \boldsymbol{l}_{\boldsymbol{i}}^{\boldsymbol{I}}, \boldsymbol{l}_{\boldsymbol{i}}^{\boldsymbol{P}}$ are labels from both label spaces $L_{I}$ and $L_{P}$ respectively. Data and regularization terms are detailed in the following sections.

\section{Data Likelihood}

The data term is defined for interconnected pairs of nodes $(i, j)$ between the two graphs (where $i \in V^{I}, j \in V^{P}$ ) and their corresponding labels $\boldsymbol{l}^{I} \in$ $L_{I}, \boldsymbol{l}^{\boldsymbol{P}} \in L_{P}$. It is encoded in the pairwise terms $e^{D} \in E_{D}$. As we described before, a plane and an in-plane deformation 2D-vector are associated with every control-point. Combining both labels, we calculate the final position of the control point $\boldsymbol{p}_{\boldsymbol{k}}$ and extract an oriented patch $\Omega_{k}$ over the plane $\pi_{k}$ (centered in $\boldsymbol{p}_{\boldsymbol{k}}$ ) from the volume $J$, so that the similarity measure $\delta$ can be calculated between that patch and the corresponding area over the $2 \mathrm{D}$ source image:

$$
e_{i, j}^{D}\left(\boldsymbol{l}_{\boldsymbol{i}}^{\boldsymbol{I}}, \boldsymbol{l}_{\boldsymbol{j}}^{\boldsymbol{P}}\right)=\int_{\Omega_{k}} \delta\left(I(\boldsymbol{x}), \pi_{k}[J](\boldsymbol{x})\right) d \boldsymbol{x} .
$$

The patch-based similarity measure $\delta$ (defined on the sub-domain $\Omega_{k}$ ) can encompass a wide choice of intensity-based measures. One of the simplest and most used similarity measures is the Sum of Absolute Differences (SAD). It is useful in the monomodal scenario, where two images of the same modality are compared. Its formulation is:

$$
e_{S A D_{i, j}}^{D}\left(\boldsymbol{l}_{\boldsymbol{i}}^{\boldsymbol{I}}, \boldsymbol{l}_{\boldsymbol{j}}^{\boldsymbol{P}}\right)=\int_{\Omega_{k}} \mid\left(I(\boldsymbol{x})-\pi_{k}[J](\boldsymbol{x}) \mid d x .\right.
$$

In multimodal scenarios, where different modalities are compared (e.g. CT with US images), statistical similarity measures such as Mutual Information (MI) are generally used since we cannot assume that corresponding objects have the same intensities in the two images. MI is defined using the joint 


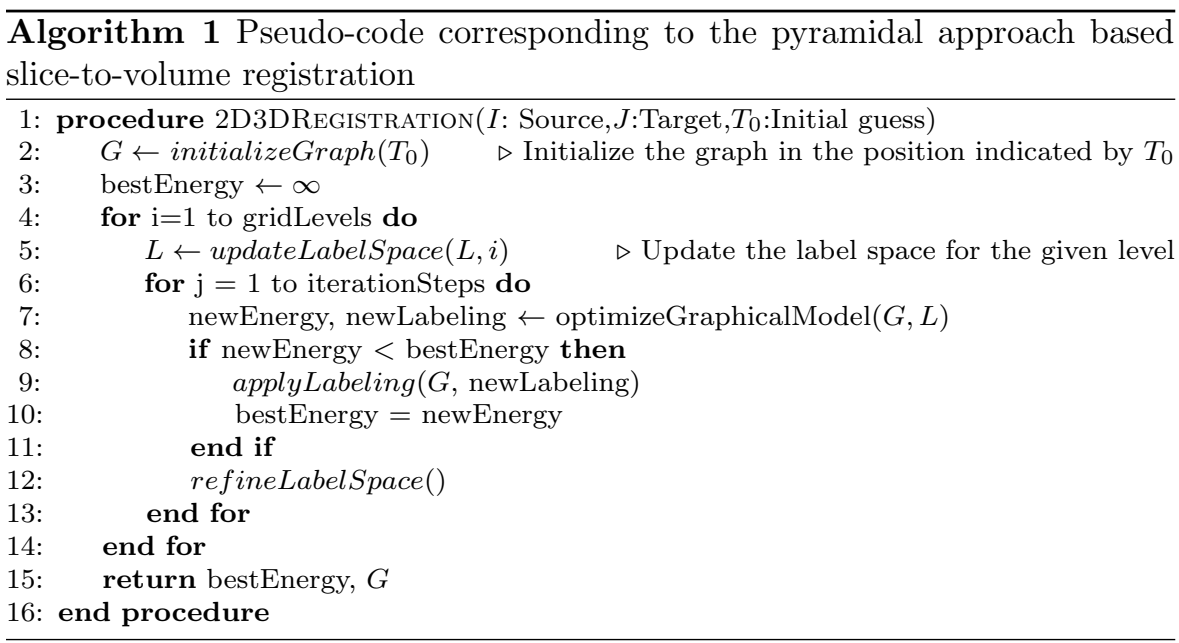

intensity distribution $p(i, j)$ and the marginal intensity distribution $p(i)$ and $p(j)$ of the images as:

$$
e_{M I_{i, j}}^{D}\left(\boldsymbol{l}_{\boldsymbol{i}}^{\boldsymbol{I}}, \boldsymbol{l}_{\boldsymbol{j}}^{\boldsymbol{P}}\right)=-\int_{\Omega_{k}} \log \frac{p\left(I(\boldsymbol{x}), \pi_{k}[J](\boldsymbol{x})\right)}{p(I(\boldsymbol{x})) p\left(\pi_{k}[J](\boldsymbol{x})\right)} d x .
$$

As we could see in the previous examples, our framework can be endowed with any similarity measure defined on two bidimensional images. In this work, we use SAD for the monomodal heart dataset and MI for the multimodal brain dataset.

\section{Regularization Terms}

We define two different regularization terms, one regularizing the plane selection and the other one the in-plane deformation. The first regularization term penalizes the average distance between the nodes $i, j \in V^{P}$ and the plane corresponding to the neighboring one. If $D_{\pi}(\boldsymbol{p})$ indicates the point-to-plane distance between the point $\boldsymbol{p}$ and the plane $\pi$, we define the regularization term $e^{P}$ as the average of these distances for two neighboring points $i, j$ and their corresponding planes:

$$
e_{i, j}^{P}\left(\boldsymbol{l}_{\boldsymbol{i}}^{\boldsymbol{P}}, \boldsymbol{l}_{\boldsymbol{j}}^{\boldsymbol{P}}\right)=\frac{1}{2}\left(D_{\pi_{j}}\left(\boldsymbol{p}_{\boldsymbol{i}}{ }^{\prime}\right)+D_{\pi_{i}}\left(\boldsymbol{p}_{\boldsymbol{j}}{ }^{\prime}\right)\right) .
$$

where $\boldsymbol{p}_{\boldsymbol{i}}{ }^{\prime}$ and $\boldsymbol{p}_{\boldsymbol{j}}{ }^{\prime}$ are the positions after applying label $\boldsymbol{l}_{\boldsymbol{i}}^{\boldsymbol{P}}, \boldsymbol{l}_{\boldsymbol{j}}^{\boldsymbol{P}}$ to $\boldsymbol{p}_{\boldsymbol{i}}, \boldsymbol{p}_{\boldsymbol{j}}$ respectively. This value is 0 when both points lie the same plane.

The second regularization term controls the in-plane deformation and is defined between nodes $i$ and $j$ included in $V_{I}$. We use a distance preserving approach which is symmetric, based on the ratio between the current position of the control points $\boldsymbol{p}_{\boldsymbol{i}}, \boldsymbol{p}_{\boldsymbol{j}}$ and their original position $\boldsymbol{p}_{\boldsymbol{o}, \boldsymbol{i}}, \boldsymbol{p}_{\boldsymbol{o}, \boldsymbol{j}}$ : 


$$
\psi_{i, j}\left(\boldsymbol{l}_{\boldsymbol{i}}^{\boldsymbol{I}}, \boldsymbol{l}_{\boldsymbol{j}}^{\boldsymbol{I}}\right)=\frac{\left\|\left(\boldsymbol{p}_{\boldsymbol{i}}+\boldsymbol{l}_{\boldsymbol{i}}^{\boldsymbol{I}}\right)-\left(\boldsymbol{p}_{\boldsymbol{j}}+\boldsymbol{l}_{\boldsymbol{j}}^{\boldsymbol{I}}\right)\right\|}{\left\|\left(\boldsymbol{p}_{o, i}\right)-\left(\boldsymbol{p}_{o, j}\right)\right\|} .
$$

Once defined $\psi_{i j}$, we need our regularizer to fulfill two conditions: first, we want it to be symmetric with respect to the displacement of the points, i.e. to penalize with the same cost whenever the control points are closer or more distant; second, we need the energy to be zero when the points are preserving distances and bigger than zero otherwise. The following regularization term fulfills both conditions for a couple of nodes $i, j \in V^{I}$ labeled with labels $\boldsymbol{l}_{\boldsymbol{i}}^{\boldsymbol{I}}, \boldsymbol{l}_{\boldsymbol{j}}^{\boldsymbol{I}}$ :

$$
e_{i, j}^{I}\left(\boldsymbol{l}_{\boldsymbol{i}}^{\boldsymbol{I}}, \boldsymbol{l}_{\boldsymbol{j}}^{\boldsymbol{I}}\right)=\left(1-\psi_{i, j}\left(\boldsymbol{l}_{\boldsymbol{i}}^{\boldsymbol{I}}, \boldsymbol{l}_{\boldsymbol{j}}^{\boldsymbol{I}}\right)\right)^{2}+\left(1-\psi_{i, j}\left(\boldsymbol{l}_{\boldsymbol{i}}^{\boldsymbol{I}}, \boldsymbol{l}_{\boldsymbol{j}}^{\boldsymbol{I}}\right)^{-1}\right)^{2} .
$$

Note that both types of pairwise terms are not sub-modular since we include the current position of the points (which can be arbitrary) in their formulation and therefore sub-modularity constraint is not fulfilled.

\section{Implementation Details}

We adopt a pyramidal approach, using different grid resolution levels, from coarse to fine spacing between the control points. For each grid resolution, some iterations of the registration algorithm are performed, choosing the best possible set for each one and updating the control point positions with this information. During the inner iterations of one grid level, the size of the displacement vectors that form the deformation label space as well as the parameter variation of the plane label space are reduced in order to improve the search space sampling. A pseudocode of the algorithm is shown in Algorithm 1.

The pairwise graphical model is optimized using the Loopy Belief Propagation algorithm (other discrete optimization algorithms can be used as well) implemented in the OpenGM2 library [12]. In [7], we used FastPD [14] instead of Loopy Belief Propagation for optimizing our pairwise model, which is among the most efficient optimization algorithms. However, due to its construction (lifting of the duality gap minimization) FastPD requires in general (towards optimizing complexity) an equal number of labels for all nodes which is an issue in our setting given the different dimensionality of the graph spaces (3d and $2 \mathrm{~d}$ ). Furthermore, while it can converge to a minimum even for nonsubmodular graphs, it is known that the quality of the linear programming (LP) relaxation is far from being satisfied and therefore the solution itself might be a very bad local minimum. Message passing methods like Loopy Belief Propagation do not inherit the computational constraints of FastPD while it is known (at least experimentally) that do good job as well even with highly non-submodular pairwise functions. 


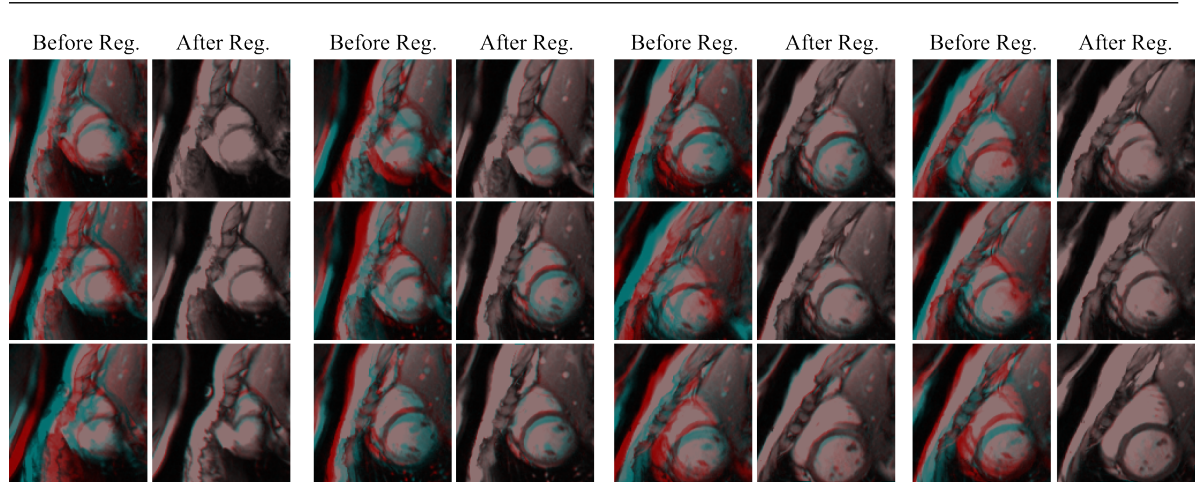

Fig. 212 registration cases of the same sequence, before and after registration. The overlapping images (in light blue we show the source image and in red the target) showed before registration corresponds to the source image and a slice taken from the volume at the initial position. The overlapping after registration corresponds to the deformed source image and the slice taken from the volume at the estimated plane position.

\section{Validation \& Results Discussion}

We validate our method in two different scenarios and we compare the results with our previous method [7]. The first one corresponds to a monomodal sequence of 2D MRI images randomly extracted from a 3D MRI temporal series of a beating heart. The second one is a multimodal brain dataset formed by 2D US images and 3D CT extracted from [20].

In order to compare both methods in a fair way, we exhaustively tested different parameter configurations (empirically for every dataset) on a grid of discretized values, and we took the best combination for each method.

\subsection{Heart Dataset}

The MRI heart dataset consists of ten sequences of twenty bidimensional MRI slices each one, that are registered with a MRI volume, giving a total of 200 registration cases. In order to generate them, as it was described in [7], we took a temporal series of $20 \mathrm{MRI}$ volumes of a beating heart, and we extracted ten random trajectories of twenty slices $I_{i}$ each one (one slice for every volume $M_{i}$ ). Starting from a random initial rotation $R_{0}=\left(R_{x_{0}}, R_{y_{0}}, R_{z_{0}}\right)$ and translation $T_{0}=\left(T_{x_{0}}, T_{y_{0}}, T_{z_{0}}\right)$, we extracted a $2 \mathrm{D}$ slice $I_{0}$ from the initial volume $M_{0}$. In every sequence, the position of slice $I_{i}$ was generated adding Gaussian noise to the position of slice $I_{i-1}$ with $\sigma_{r}=3^{\circ}$ and $\sigma_{t}=5 \mathrm{~mm}$ to every translation $\left(T_{x}, T_{y}, T_{z}\right)$ and rotation $\left(R_{x}, R_{y}, R_{z}\right)$ parameters respectively. It gives maximum distances of about $25 \mathrm{~mm}$ between the current and its succeeding slice. The MRI resolution was $192 \times 192 \times 11$ and the voxel size was $1.25 \times 1.25 \times 8 \mathrm{~mm}^{3}$.

For every sequence, we initialize the registration adding the same noise (with the same parameters than before) to the ground truth. During the reg- 


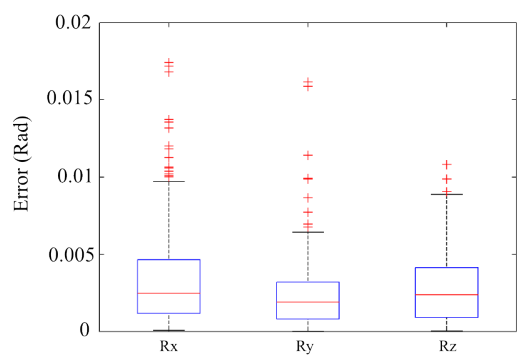

(a)

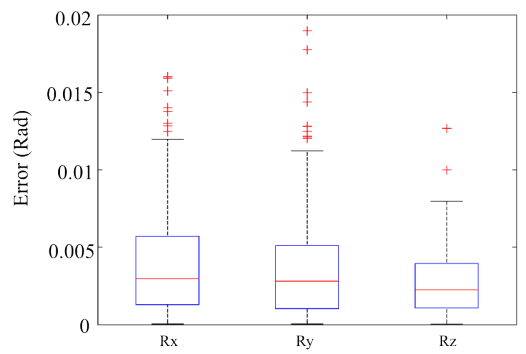

(c)

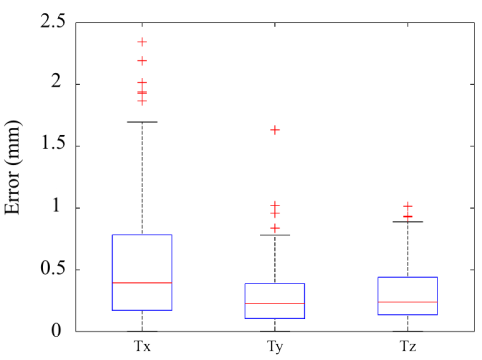

(b)

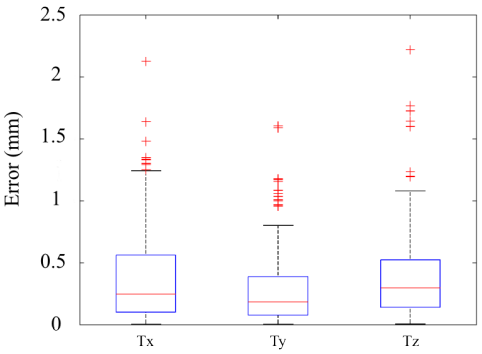

(d)

Fig. 3 Comparison of the error estimation for plane parameters $\left(R_{x}, R_{y}, R_{z}\right)$ and $\left(T_{x}\right.$ $T_{y}, T_{z}$ ) for our decoupled method (figures (a) and (b)) and the overparameterized approach presented by [7] (figures (c) and (d)). For presentation clarity, three outliers between 0.02 and $0.05 \mathrm{rad}$ as well as one at $4 \mathrm{~mm}$ have been removed at Figures (c) and (b) respectively.

istration process, given two consecutive slices of the same sequence, the estimated transformation for slice $I_{i}$ was used as initialization for the registration of slice $I_{i+1}$.

\begin{tabular}{|c|c|c|c|c|c|c|c|}
\hline & & $R_{x}$ & $R_{y}$ & $R_{z}$ & $T_{x}$ & $T_{y}$ & $T_{z}$ \\
\hline \multirow{2}{*}{ Decoupled Method } & Mean & 0.0036 & 0.0024 & 0.0029 & 0.5403 & 0.2713 & 0.2966 \\
\cline { 2 - 8 } & $\mathrm{SD}$ & 0.0034 & 0.0024 & 0.0024 & 0.4914 & 0.2296 & 0.2236 \\
\hline \multirow{2}{*}{ Overparameterized Method [7] } & Mean & 0.0051 & 0.0051 & 0.0031 & 0.4164 & 0.2874 & 0.4847 \\
\cline { 2 - 8 } & $\mathrm{SD}$ & 0.0122 & 0.0134 & 0.0051 & 0.4720 & 0.2976 & 1.1546 \\
\hline
\end{tabular}

Table 1 Error estimation for plane parameters $\left(R_{x}, R_{y}, R_{z}\right)$ and $\left(T_{x}, T_{y}, T_{z}\right)$ for our decoupled method and the previous overparameterized approach presented in [7].

Figure 2 shows the overlapping between the source image and the corresponding target plane, before and after registration, for 12 cases of one sequence. As we can observe in a qualitative way, the overlapping increases after registration.

Figure 3 compares our results in a quantitative way with the ones obtained using our previous method. We measure the error between the estimated transformation parameters and the ground truth. The mean error was (0.0036, $0.0024,0.0029) \mathrm{rad}$ for rotation and $(0.5403,0.2713,0.2966) \mathrm{mm}$ for translation parameters, with a standard deviation of $(0.0034,0.0024,0.0024) \mathrm{rad}$ 
and $(0.4914,0.2296,0.2236) \mathrm{mm}$ respectively. The average running time was around 60 seconds for every registration case. Using the method presented in [7], we obtained $(0.0051,0.0051,0.0031) \mathrm{rad}$ and $(0.4164,0.2874,0.4847) \mathrm{mm}$ for rotation and translation parameters error, and standard deviation equal to $(0.0122,0.0134,0.0051) \mathrm{rad}$ and $(0.4720,0.2976,1.1546) \mathrm{mm}$. Results are presented in Table 1. Every registration case took around 220 seconds (almost 3.5 times more than our method). As we can see, the quality of the results was preserved (and improved in some cases) while the computational time was reduced approximately 3.5 times (keeping equivalent grid and label space sizes, sampling patch size and number of algorithm iterations).

Validation of in-plane deformation was performed over 20 registration cases, deforming an initial segmentation of the left endocardium using the estimated deformation field $T_{D_{i}}$. We measure the average DICE coefficient between the segmentations, before and after deforming the initial one, to measure the impact of the deformation in the registration process. The average DICE before deformation was 0.858 and after registration was 0.907 , showing that our method can capture in-plane deformations and select the correct plane at the same time.

Common parameters used for both methods were 3 grid levels, 5 iterations per level, initial control point distance of $40 \mathrm{~mm}$ and minimum sampling patch size of $20 \mathrm{~mm}$. In case of the decoupled model we use $\gamma=1, \beta=0.2, \alpha=0.8$, 41 labels in the plane label space and 91 labels in the deformations label space. In case of the overparameterized model we use 13122 labels and $\alpha=0.9$ (for a complete understanding of these parameters refer to [7]). We run the experiments on an Intel Xeon W3670 with 6 Cores, 64bits and 16GB of RAM.

\subsection{Brain Dataset}

The brain dataset consists of a pre-operative brain MRI volume (voxel size of $0.5 \times 0.5 \times 0.5 \mathrm{~mm}^{3}$ and resolution of $394 \times 466 \times 378$ voxels) and 6 series of 9 US images extracted from the patient 01 of the database MNI BITE presented in [20]. The size of the US images was $48 \times 38 \mathrm{~mm}$ and the pixel resolution $0.3 \times 0.3 \mathrm{~mm}$. The ventricles were manually segmented by specialists in both modalities and used to calculate DICE coefficient and Contour Mean Distance (CMD) to evaluate and compare the quality of the results. Initializations were done following the same methodology that we described for the Heart Dataset (Section 3.1).

Figure 4 summarizes the average DICE and CMD coefficients for each series. It shows that, using our decoupled method, the mean DICE increases after the registration process an average of 0.0405 , a little bit more than the 0.0380 obtained with [7] method. Regarding the CMD, the average decrement for our method is $0.3654 \mathrm{~mm}$ while for the other one is $0.3943 \mathrm{~mm}$. Even if our new method performs better in average, we can observe that results are almost equivalent in terms of DICE and CMD. However, there is a big difference in terms of computing time: while our method is taking around 3 min per reg- 

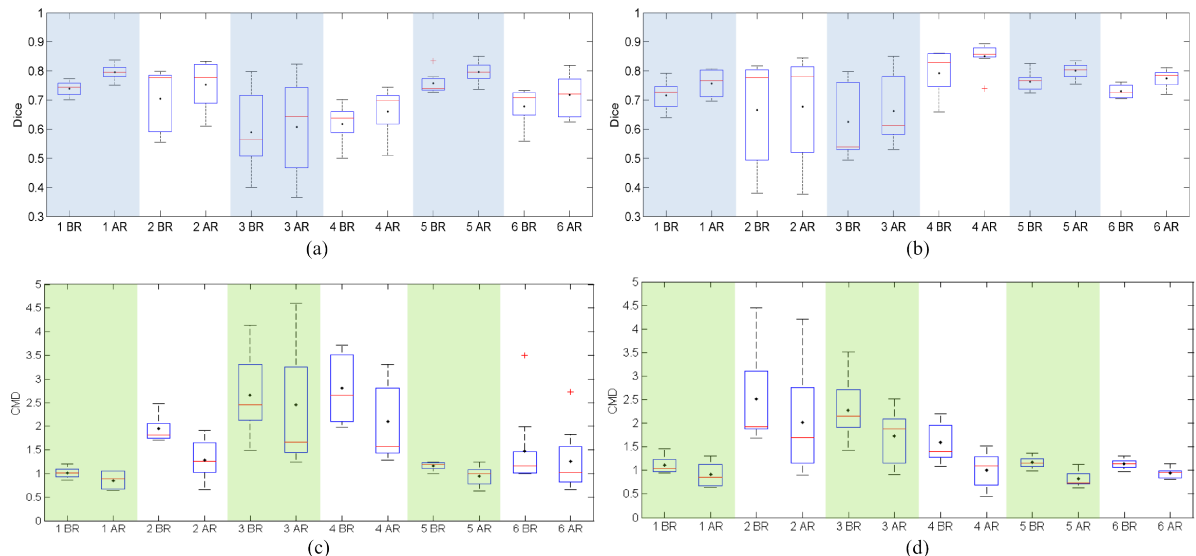

Fig. 4 The figures show a quantitative comparison of the two methods, before (BR) and after (AR) registration for the 6 sequences of brain data. Figures (a) and (c) show results for our decoupled method (DICE and CMD respectively) while figures (b) and (d) show results for the overparameterized approach presented in [7] (DICE and CMD respectively).

istration case, the overparameterized method takes around 10 min running in the same computer using the same configuration. To perform the experiments with both methods, we used the same configuration given by 3 grid levels, initial control point distance of $8 \mathrm{~mm}, 4$ iterations per level and minimum sampling patch size of $13 \mathrm{~mm}$. In case of the decoupled model, we set $\gamma=1$, $\beta=0.05, \alpha=0.2,41$ labels in the plane label space and 91 labels in the deformations label space. For the overparameterized method we set $\alpha=0.8$ and 6174 labels. We run the experiments in the same Intel Xeon W3670 with 6 Cores, 64bits and 16GB of RAM used for the heart dataset.

\subsection{Discussion \& Comparison With Other Methods}

As we have shown, our method is able to achieve state of the art results while decreasing the computational time when we compare to another MRF based method (namely [7]). In the monomodal case we reduce it from around $3.5 \mathrm{~min}$ to $1 \mathrm{~min}$ while in the multimodal one we go from $10 \mathrm{~min}$ to $3 \mathrm{~min}$, giving a time factor reduction of about 3 times.

The main strength of the proposed formulation is the linear complexity of the inference process with respect to the product of the label spaces. This allows to go even further for challenging cases (brain tumor removal) where precision is required to substantially increase the label space. This is not the case for the approach presented in [7] due to the complexity of the label space.

An interesting point to discuss about is the 5 -fold improvement in the standar deviation error of parameter $T_{z}$ that we obtain with the new method. In [7], the justification for the poor performance of the method when estimating $T_{z}$ was told to be that image resolution in $\mathrm{z}$ axis was lower than in $\mathrm{x}$ and $\mathrm{y}$. We 

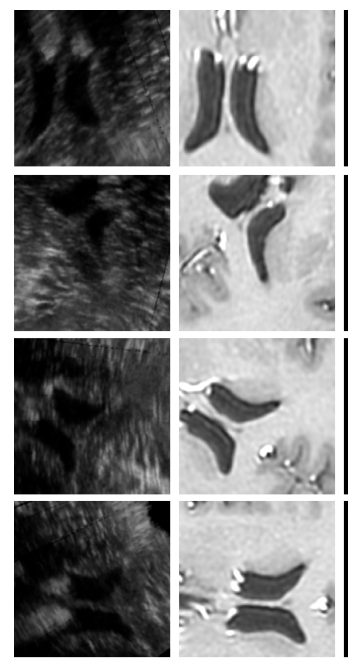

(a)
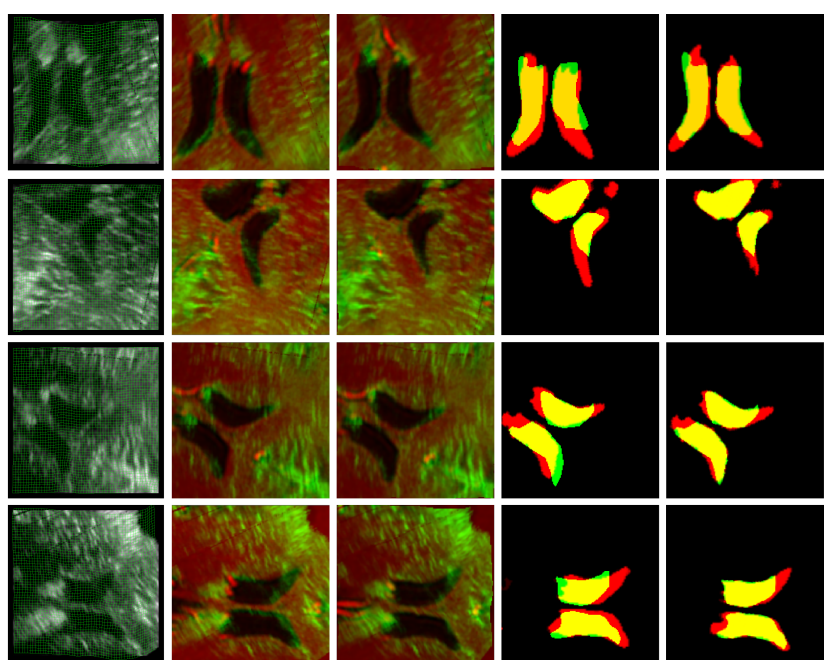

(c)
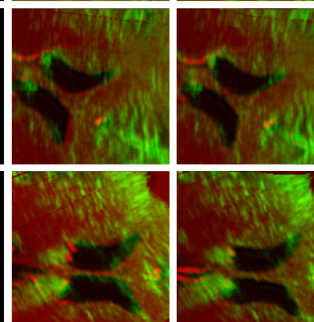

(d)

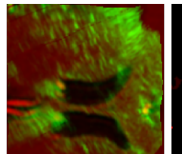

(e)

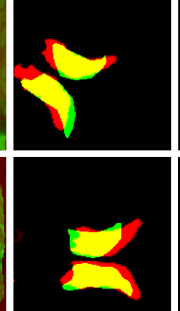

(f)

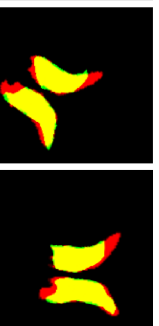

(g)

Fig. 5 Results for one slice from four of the six brain sequences (each row correspond to a different sequence). (a) Source 2D Ultrasound image. (b) Slice extracted from the MRI corresponding to the initial position of the plane. (c) Deformed source image overlapped with the estimated deformation field. (d) Blending between initial images (US and corresponding MRI slice). (e) Blending between final images (Deformed US image and estimated MRI slice). (f) Overlapping between initial segmentations. (g) Overlapping between segmentations after registration.

think that the new algorithm is less sensitive to image resolution anisotropy mainly because of the different way we explore the plane-selection label space by allowing a deeper exploration when decoupling it without exponentially increasing the amount of labels.

It is important to remark that both, the decoupled and overparameterized methods, are highly dependent on the initialization given for the first slice of the sequence. Since these algorithms optimize the energy based on a limited search space (determined by the label space), if the solution is not reachable from the intial position using the current label space, the algorithm will fail. Another factor that is crucial for the success of the algorithm is the similarity measure used to decide whether or not two patches coming from different images correspond to the same anatomical structure. The study of different similarity measures is outside the scope of this paper; however, note that in order to use the method in other image modalities, it will be necesary to choose an accurate similarity measure and calibrate the parameters accordingly.

Comparison with other methods in the field of slice-to-volume registration is a complicated task, mainly because of the lack of public datasets. Here we include some of the results reported by other state of the art methods for their own datasets, in terms of accuracy and/or performance. In [10] for example, authors report a mean Target Registration Error (TRE) lower than $1 \mathrm{~mm}$ when estimating rigid transformations in a monomodal MRI dataset of 
prostate images (for a pixel size of $1.5 \times 1.5 \times 3 \mathrm{~mm}$ ). Random initializations were generated by modifying the ground truth position with displacements of $10 \mathrm{~mm}$ and rotations of 10deg maximum. The Matlab implementation of their algorithm took between $36 \mathrm{sec}$ and $107 \mathrm{sec}$ depending on the algorithm configuration. In [23], authors tested on a multimodal dataset formed by 2D ultrasound and CT volumes of the heart. They report errors around $1.56 \pm$ $0.78 \mathrm{~mm}$ when estimating rigid transformations on CT images with $0.6 \mathrm{~mm}$ isotropic resolution, using initializations with uniformly random shifts in the range -5 to $5 \mathrm{~mm}$. They achieve quasi real time performance with execution times around $4 \mathrm{sec}$. Another interesting example to compare with is the multislice to volume registration case that tackles [25] applying it to MRI-guided transperineal prostate biopsy. Authors report that deformable registrations were accurate to within $2 \mathrm{~mm}$ in images with a slice spacing of $3.6 \mathrm{~mm}$. The execution time for the complete deformable registration algorithm is about $30 \mathrm{sec}$. Even if it is not possible to do a fair comparison mainly because of the lack of standard benchmarks, by observing these examples we can clearly remark that our results are in the state of the art level. Moreover, visual assessment on the obtained results seems to confirm that these are satisfactory in the context of a clinical setting.

In terms of complexity, it is interesting to remark the difference with respect to our previous method. The optimization complexity/difficulty heavily depends on the maximum number of label combinations that the pairwise cliques can take (this is the bottle neck for most optimization algorithms). In this perspective, the complexity of the overparameterized model is given by $O\left(|L|^{2}\right.$ ), where $|L|$ is the cardinality (number of labels) of the label space. In our new approach, we introduce two label spaces $L_{1}$ and $L_{2}$ that decouple the previous one. To give an idea about the reduction in the complexity of our new model, let us say that $|L|=\left|L_{1} \cdot L_{2}\right|$. Because of the way in which we construct our decoupled graph (as it is indicated in Figure 1), it is straightforward to show that the complexity of the new model reduces now to $O\left(\max \left(\left|L_{1}\right|,\left|L_{2}\right|\right)^{2}\right)$. Therefore, because of the decoupling strategy, the complexity of the model reduces to the square of the cardinality of the biggest label space (instead of being quadratic in the cardinalities of the joint space), with a slight increase of the graphical model connectivity. Consequently, while the number of nodes augment linearly, the number of labels is decreased in a quadratic order.

\section{Conclusions}

We presented a new method to perform slice-to-volume registration based on a decoupled model that associates two local graphs to the plane selection and the in-plane deformations while imposing consistency through direct connections between the corresponding nodes. In order to solve this problem, we seek the plane and the in-plane deformation that best matches our energy function. It is important to remark that we just look for the in-plane deformations given the nature of the problems we are trying to solve (mainly image fusion for 
IGS), where it is not useful to find out-of-the-plane deformations at least for visualization purposes, even if they can exist.

As we have shown in the previous section, our method achieves state of the art results while decreasing substantially the time of computation when it is compared to our previous MRF based method that uses a unique high dimensional label space [7]. It confirms our initial hypothesis, meaning that decoupling the graphical model and labeling it using two lower dimensional label spaces, we can achieve the same results while reducing the complexity of our method.

We have also shown that the method is robust with respect to the type of images we are registering. Since slice-to-volume registration has multiple applications, other problems are under investigation (it should be noted that such a task is complex due to the complete absences of public ground truth). To this end, two clinical scenarios are currently under investigation, the first refers to liver tumor resection guidance, while the second to US guidance during prostate biopsy through fusion of intra-operative ultrasound and preoperative CT/MR.

In order to improve the quality of the results, specially in multimodal cases, feature engineering must be considered. Future work includes adapting and using features specifically designed for multimodal registration such as the $L C^{2}$ presented in [8] and the MIND descriptor presented in [2]. Furthermore, energy regularizers inspired on precise biophysical modeling and tissue properties could lead to accuracy improvements as well. The underlying idea is to adapt the "smoothness" constraint of the deformation model by explicitly taking into account organ specific motion/deformation constraints like for example in the context of liver biopsies or brain tumor ablation.

Finally, we are investigating new methods to improve the parameters estimation procedure. Energy parameters estimation based on machine learning techniques [13] have to be considered as a future work if we want to exploit at the maximum level the potential of the proposed method.

\section{Funding}

This research was partially supported by European Research Council Starting Grant Diocles (ERC-STG-259112).

\section{Conflict of Interest}

The authors declare that they have no conflict of interest.

\section{Ethical approval}

- This article does not contain any studies with animals performed by any of the authors. 
- All procedures performed in studies involving human participants were in accordance with the ethical standards of the institutional and/or national research committee and with the 1964 Helsinki declaration and its later amendments or comparable ethical standards.

\section{References}

1. Baker, S., Scharstein, D., Lewis, J., Roth, S., Black, M.J., Szeliski, R.: A database and evaluation methodology for optical flow. International Journal of Computer Vision 92(1), 1-31 (2011)

2. Bardera, A., Feixas, M., Boada, I., Sbert, M.: High-dimensional normalized mutual information for image registration using random lines. In: J. Pluim, B. Likar, F. Gerritsen (eds.) Biomedical Image Registration, Lecture Notes in Computer Science, vol. 4057, pp. 264-271. Springer Berlin Heidelberg (2006)

3. Birkfellner, W., Figl, M., Kettenbach, J., Hummel, J., Homolka, P., Schernthaner, R., Nau, T., Bergmann, H.: Rigid 2D/3D slice-to-volume registration and its application on fluoroscopic CT images. Medical Physics 34(1), 246 (2007). DOI 10.1118/1.2401661

4. Birkfellner, W., Hummel, J., Wilson, E., Cleary, K.: Tracking devices. In: Image-Guided Interventions, pp. 23-44. Springer (2008)

5. Chandler, A.G., Pinder, R.J., Netsch, T., Schnabel, J.A., Hawkes, D.J., Hill, D.L., Razavi, R.: Correction of misaligned slices in multi-slice mr cardiac examinations by using slice-to-volume registration. In: Journal of Cardiovascular Magnetic Resonance2008, 10:13 (2008)

6. Dalvi, R., Abugharbieh, R.: Fast feature based multi slice to volume registration using phase congruency. In: Engineering in Medicine and Biology Society, 2008. EMBS 2008. 30th Annual International Conference of the IEEE, pp. 5390-5393. IEEE (2008)

7. Ferrante, E., Paragios, N.: Non-rigid 2d-3d medical image registration using markov random fields. In: Medical Image Computing and Computer-Assisted InterventionMICCAI 2013, pp. 163-170. Springer (2013)

8. Fuerst, B., Wein, W., Muller, M., Navab, N.: Automatic ultrasoundmri registration for neurosurgery using the 2d and 3d lc2 metric. Medical Image Analysis 18(8), $1312-$ 1319 (2014). Special Issue on the 2013 Conference on Medical Image Computing and Computer Assisted Intervention

9. Geman, S., Geman, D.: Stochastic relaxation, gibbs distributions, and the bayesian restoration of images. Pattern Analysis and Machine Intelligence, IEEE Transactions on (6), 721-741 (1984)

10. Gill, S., Abolmaesumi, P., Vikal, S., Mousavi, P., Fichtinger, G.: Intraoperative prostate tracking with slice-to-volume registration in mri pp. 154-158 (2008)

11. Glocker, B., Sotiras, A., Komodakis, N., Paragios, N.: Deformable medical image registration: setting the state of the art with discrete methods. Annu Rev Biomed Eng 13, 219-244 (2011). DOI 10.1146/annurev-bioeng-071910-124649

12. Kappes, J.H., Andres, B., Hamprecht, F.A., Schnörr, C., Nowozin, S., Batra, D., Kim, S., Kausler, B.X., Lellmann, J., Komodakis, N., Rother, C.: A comparative study of modern inference techniques for discrete energy minimization problem (2013)

13. Komodakis, N.: Efficient training for pairwise or higher order crfs via dual decomposition. In: CVPR, pp. 1841-1848 (2011)

14. Komodakis, N., Tziritas, G., Paragios, N.: Fast, approximately optimal solutions for single and dynamic mrfs. In: Computer Vision and Pattern Recognition, 2007. CVPR'07. IEEE Conference on, pp. 1-8. IEEE (2007)

15. Kotsas, P., Dodd, T.: A review of methods for $2 \mathrm{~d} / 3 \mathrm{~d}$ registration. WASET Conference Paris 14-16 November (2011)

16. Lee, K., Kwon, D., Yun, I., Lee, S.: Deformable 3d volume registration using efficient mrfs model with decomposed nodes. In: British Machine Vision Conference, pp. 1-10 (2008) 
17. Mahapatra, D., Sun, Y.: Nonrigid registration of dynamic renal mr images using a saliency based mrf model. Medical Image Computing and Computer-Assisted Intervention-MICCAI 2008 pp. 771-779 (2008)

18. Markelj, P., Tomaževič, D., Likar, B., Pernuš, F.: A review of $3 \mathrm{~d} / 2 \mathrm{~d}$ registration methods for image-guided interventions. Medical image analysis (2012)

19. Marks, L., Young, S., Natarajan, S.: Mri-ultrasound fusion for guidance of targeted prostate biopsy. Current opinion in urology 23(1), 43 (2013)

20. Mercier, L., Del Maestro, R.F., Petrecca, K., Araujo, D., Haegelen, C., Collins, D.L.: Online database of clinical $\mathrm{mr}$ and ultrasound images of brain tumors. Medical Physics 39, 3253 (2012)

21. Osechinskiy, S., Kruggel, F.: Slice-to-volume nonrigid registration of histological sections to $\mathrm{mr}$ images of the human brain. Anatomy Research International 2011 (2010)

22. Penney, G., Blackall, J., Hayashi, D., Sabharwal, T., Adam, A., Hawkes, D.: Overview of an ultrasound to ct or $\mathrm{mr}$ registration system for use in thermal ablation of liver metastases. In: Proc. Medical Image Understanding and Analysis, vol. 1, p. 6568. Citeseer (2001)

23. San José Estépar, R., Westin, C., Vosburgh, K.: Towards real time 2d to 3d registration for ultrasound-guided endoscopic and laparoscopic procedures. International journal of computer assisted radiology and surgery 4(6), 549-560 (2009)

24. Shekhovtsov, A., Kovtun, I., Hlaváč, V.: Efficient mrf deformation model for nonrigid image matching. Comput. Vis. Image Underst. 112(1), 91-99 (2008). DOI 10.1016/j.cviu.2008.06.006. URL http://dx.doi.org/10.1016/j.cviu.2008.06.006

25. Xu, H., Lasso, A., Fedorov, A., Tuncali, K., Tempany, C., Fichtinger, G.: Multi-slice-tovolume registration for mri-guided transperineal prostate biopsy. International journal of computer assisted radiology and surgery pp. 1-10 (2014)

26. Zikic, D., Glocker, B., Kutter, O., Groher, M., Komodakis, N., Kamen, A., Paragios, N., Navab, N.: Linear intensity-based image registration by markov random fields and discrete optimization. Medical Image Analysis 14(4), 550-562 (2010). DOI 10.1016/j.media.2010.04.003. URL http://dx.doi.org/10.1016/j.media.2010.04.003 